\title{
The effects of surgical treatment with chondroblastoma in children and adolescents in open epiphyseal plate of long bones
}

Yan Xiong ${ }^{\dagger}$, Yun Lang ${ }^{\dagger}$, Zeping Yu, Hongyuan Liu, Xiang Fang, Chongqi Tu and Hong Duan ${ }^{*}$

\begin{abstract}
Background: Chondroblastoma is a rare benign cartilaginous tumor, which primarily occurs in children and adolescents. Chondroblastoma commonly originates in the epiphyseal plate of long bones. An aggressive curettage treatment is recommended to manage lesion, which may jeopardize an open epiphyseal plate and result in limb shortening and deformity as the limb grows and develops. The purpose is to observe surgical effects of chondroblastoma on open epiphyseal plate of long bones in children and adolescents and explore influences on limb growth and development.
\end{abstract}

Methods: We retrospectively reviewed 18 cases of long bone chondroblastoma with open epiphyseal growth plate during March 2004 to October 2010 in our center. Seven females and 11 males with mean age of $11.6 \pm 2.0$ years old (8-15 years) were included. Patients, who suffered from trauma and pathological fracture of the epiphyseal plate or congenital diseases such as poliomyelitis, congenital dementia, and cartilage malnutrition, were excluded. All patients were treated with meticulous intralesional curettage and inactivity with alcohol followed by bone grafts. All cases were followed up $8.2 \pm 1.7$ years (5-11.5 years).

Results: All had no local recurrence and distance metastasis. The length of the affected limb was short, $18.47 \pm 7.22 \mathrm{~mm}$ $(1.5-30 \mathrm{~mm})$. There was no obvious relativity with tumor activity $(P=0.061)$. Meanwhile, there were obvious relativity with the greatest dimension of the lesion (TGD) $(P=0.003)$, the vertical dimension between edge of lesion and epiphyseal line (TVD) $(P=0.010)$, and area ratio of lesion to local epiphysis (lesion/growth plate) $(P=0.015)$. The MSTS93 (Revised Musculoskeletal Tumor Society Rating Scale 93) and SF-36 (Medical Outcomes Study 36-Item Short-Form Health Survey) had been significantly improved $(P<0.01)$.

Conclusion: Managing of chondroblastoma located in open epiphyseal plate of a long bone with meticulous curettage, inactivity, and bone grafts can control tumor progression and recurrence effectively. Meanwhile, early detection and prompt surgical treatment intervention, which reduced significantly the tumor to influence limb growth and development, get encouraging limb function.

Trial registration: This is a retrospective study, which was not registered in any trial registry.

Keywords: Chondroblastoma, Epiphysis, Open epiphyseal plate, Limb-length, Children and adolescents

\footnotetext{
*Correspondence: duanhong1970@126.com

${ }^{\dagger}$ Equal contributors

Department of Orthopedics, West China Hospital, Sichuan University, No 37

Guo Xue Lane, Wuhou District, 610041 Chengdu, Sichuan, People's Republic

of China
} 


\section{Background}

Chondroblastoma is a rare benign bone tumor with an incidence of $9 \%$ in benign bone tumors [1]. Chondroblastoma commonly occurs in 10 25-year-old person. Previous studies revealed that it originated from chondroblasts, and entity was classified as a benign chondroblastoma of bone [2-5]. The chondroblastoma is typically located in the epiphysis of a long bone and less often in the apophysis [6-9]. Due to active and aggressive characteristics, chondroblastoma invade and destroy regularly adjacent tissues as tumor progression [6-9]. Meanwhile, knee, hip, and shoulder joints frequently are involved [9]. Common presenting symptoms include rest or activity pain and local tenderness, followed by swelling and limited motion of neighboring joints [9]. In serious cases, neighboring nerves and vascular joints are compressed and invaded. Thus, early detection and prompt treatment intervention are significant.

Surgery is recommended for treatment with chondroblastoma mainly [1, 9, 10]. The gold standard for surgical treatment is accurate and meticulous intralesional curettage with or without local adjuvant therapy followed by bone grafting [10]. The rate of recurrence after those procedures has been reported between 10 and 36\% [3, 6, 7]. Some complications can be observed after surgical therapy due to involvement of the epiphysis, such as limb-length discrepancy and articular deformity with a frequency of $7-50 \%[9,10]$.

To our knowledge, there were rare study series exclusively focusing on limb length of chondroblastoma after surgery in young patients with open epiphyseal growth plate $[9,10]$. Epidemiologic characteristics and predictors of involved limb length of chondroblastoma during growth were not known. The objectives of the present retrospective study were to observe the effects of removed epiphyseal chondroblastoma during growth in 18 patients who were children and adolescents and to identify what factors might influence limb growth and development after surgical treatment.

\section{Methods}

\section{Inclusion criteria}

All patients with open epiphyseal growth plate of long bone chondroblastoma were accepted during March 2004 to October 2010 in our center. The chondroblastoma was confirmed by pathology. All patients received the first tumor resection. Operation agreement and rehabilitation protocol were signed.

\section{Exclusion criteria}

The patient with closed epiphyseal plate was excluded. Trauma and pathological fracture of epiphyseal plate was not involved. Patients who suffered from poliomyelitis, congenital dementia, cretinism, cerebral palsy, and cartilage malnutrition were not unaccepted.

\section{Clinical data}

We performed a retrospective study of 18 children and adolescents with chondroblastoma in our hospital from March 2004 to October 2010. The diagnosis was based on recognized image data and histological criteria including intraoperative frozen pathological and postoperative paraffin tissue biopsy. The general information is described in Table 1. All operations were performed in West China Hospital, Sichuan University by two senior surgeons (Drs. Chongqi Tu and Hong Duan). This study has been approved by the Ethics committee at West China Hospital of Sichuan University.

All patients underwent chest radiography and radionuclide bone imaging on presentation as a screening procedure for metastatic disease, and no metastatic disease occurred. The status of adjacent epiphyseal plate was determined by radiographs. An epiphyseal plate was classified as open if a wide and clearly defined radiolucency was apparent. Meanwhile, an epiphyseal plate was closed if an epiphyseal scar was present [10]. The lesion activity was classified as latent, active, and aggressive using of the system of Patrick C.et al. [11]. All 18 patients in our research had open epiphyseal plate according to this criterion.

\section{Measuring methods}

We measured the length of each long bone according to X-ray on computer (Syngo version V35; Siemens Medical Systems, Erlangen, Germany) (Fig. 1). All lesions were nearly circular in the coronal plane in CT (computer tomography) or MRI (magnetic resonance imaging). Therefore, we measured the diameter of the biggest circular as $2 \mathrm{r}$ (radius of the lesion) and the minor and major axes of the oval as 2a (major axes) and $2 \mathrm{~b}$ (major axes). Focus/epiphyseal plate as the area proportion of the circular and oval can be calculated from the following formula (focus/epiphyseal plate $=\pi r^{2} / \pi a b$, Fig. 2). The greatest dimension of the lesion (TGD) was measured according to MRI in the axial, sagittal, or crown plane (Fig. 3). The vertical dimension between the edge of lesion and epiphyseal line (TVD) was from the edge of the lesion reverse adjacent joint to the epiphyseal line in vertical plane of sagittal or crown MRI plane. The epiphyseal line was the flag line. The negative value indicated that the lesion damaged the epiphyseal line to involve the metaphyseal (Fig. 4). 
Table 1 Details of 18 patients

\begin{tabular}{|c|c|c|c|c|c|c|c|c|c|c|}
\hline No & Age (years) & Sex & Location & Tumor activity & Tumor size $\left(\mathrm{mm}^{3}\right)$ & $\mathrm{TGD}(\mathrm{mm})$ & $\mathrm{TVD}(\mathrm{mm})$ & Focus/growth plate & Follow-up (years) & Shorten $(\mathrm{mm})$ \\
\hline 1 & 11 & $\mathrm{~F}$ & Proximal tibia & Active & $35 \times 50 \times 50$ & 50 & -20 & $25 / 50(50 \%)$ & 9 & 30 \\
\hline 2 & 13 & M & Proximal tibia & Aggressive & $30 \times 40 \times 55$ & 55 & 3 & 15/40(38\%) & 7 & 28 \\
\hline 3 & 11 & $\mathrm{~F}$ & Proximal tibia & Active & $10 \times 10 \times 13$ & 13 & 4 & $12 / 52(23 \%)$ & 8.4 & 14 \\
\hline 4 & 12 & $\mathrm{~F}$ & Distal femur & Active & $20 \times 23 \times 26$ & 26 & 3 & $15 / 55(27 \%)$ & 8.5 & 14 \\
\hline 5 & 14 & M & Proximal humerus & Active & $40 \times 40 \times 50$ & 40 & 0 & 40/50(80\%) & 8.3 & 15 \\
\hline 6 & 11 & $\mathrm{~F}$ & Distal femur & Active & $15 \times 15 \times 20$ & 15 & 10 & $13 / 53(25 \%)$ & 10 & 12 \\
\hline 7 & 14 & M & Proximal tibia & Active & $10 \times 20 \times 25$ & 20 & 3 & 20/62(32\%) & 7 & 18 \\
\hline 8 & 10 & M & Distal femur & Aggressive & $24 \times 26 \times 28$ & 28 & -4 & 26/68(38\%) & 10 & 23 \\
\hline 9 & 10 & M & Proximal humerus & Aggressive & $35 \times 40 \times 50$ & 50 & -20 & 25/45(56\%) & 6.8 & 24 \\
\hline 10 & 15 & M & Proximal tibia & Active & $35 \times 40 \times 50$ & 50 & -15 & $25 / 50(50 \%)$ & 9.5 & 20 \\
\hline 11 & 8 & $\mathrm{~F}$ & Proximal tibia & Active & $20 \times 30 \times 35$ & 35 & -22 & 23/50(46\%) & 11.5 & 30 \\
\hline 12 & 9 & M & Proximal humerus & Aggressive & $35 \times 35 \times 40$ & 40 & -15 & 9/40(23\%) & 8.4 & 24 \\
\hline 13 & 12 & $\mathrm{~F}$ & Proximal femur & Active & $20 \times 25 \times 30$ & 30 & -5 & 10/43(23\%) & 7.5 & 13 \\
\hline 14 & 13 & M & Proximal femur & Active & $25 \times 25 \times 28$ & 28 & 2 & $12 / 46(26 \%)$ & 9.7 & 14 \\
\hline 15 & 11 & M & Proximal humerus & Active & $20 \times 25 \times 35$ & 20 & -7 & 20/50(40\%) & 7 & 15 \\
\hline 16 & 14 & M & Proximal humerus & Active & $10 \times 15 \times 23$ & 23 & -4 & 15/35(43\%) & 7.8 & 19 \\
\hline 17 & 13 & M & Proximal tibia & Active & $10 \times 12 \times 14$ & 14 & 0 & $7 / 35(20 \%)$ & 5.5 & 1.5 \\
\hline 18 & 9 & $\mathrm{~F}$ & Proximal humerus & Latent & $10 \times 10 \times 12$ & 12 & 5 & 9/37(24\%) & 5 & 18 \\
\hline
\end{tabular}

The negative value indicates that the lesion involves epiphyseal plate; focus/growth plate (\%), the largest area of the focus/lesion epiphyseal plate area Abbreviations: TGD the greatest dimension of lesion, TVD the vertical dimension between edge of lesion and epiphyseal line
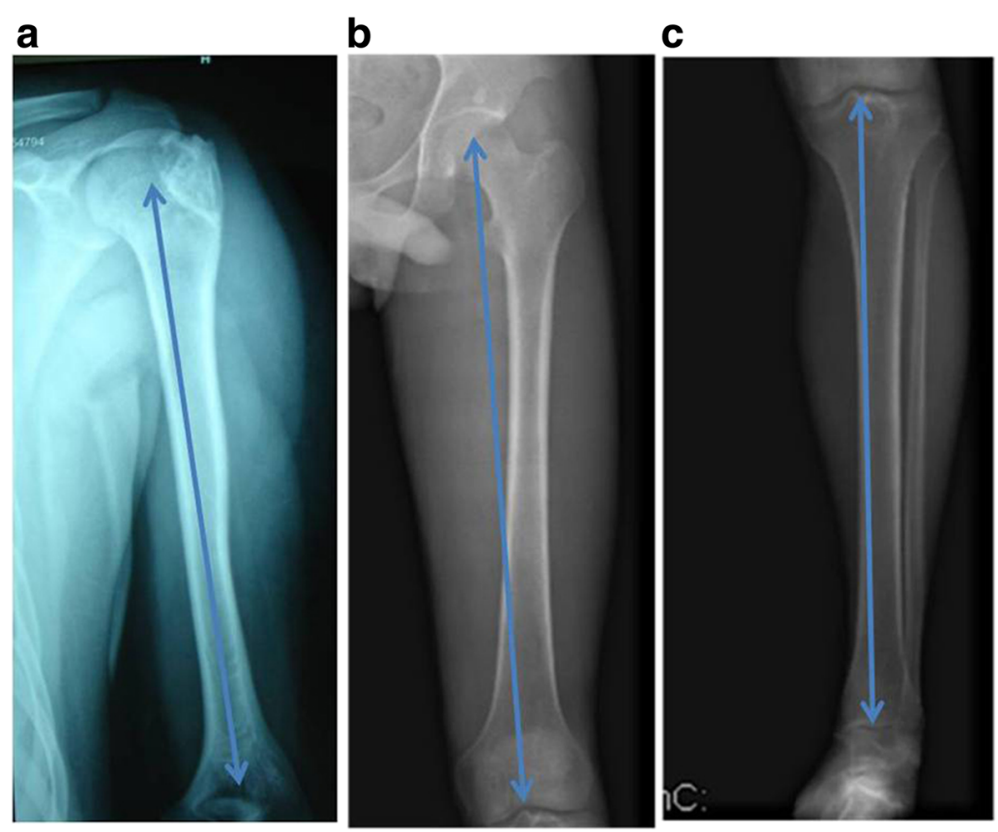

Fig. 1 a-c The method of measuring length of the bone (the arrow straight line shows), humerus, from the humeral head midpoint to the midpoint of the medial and lateral condyle. The femur, from the midpoint of the femoral head to the midpoint of the medial and lateral condyle. The tibia, from the tibial plateau midpoint to within the lateral midpoint of the distal tibia 


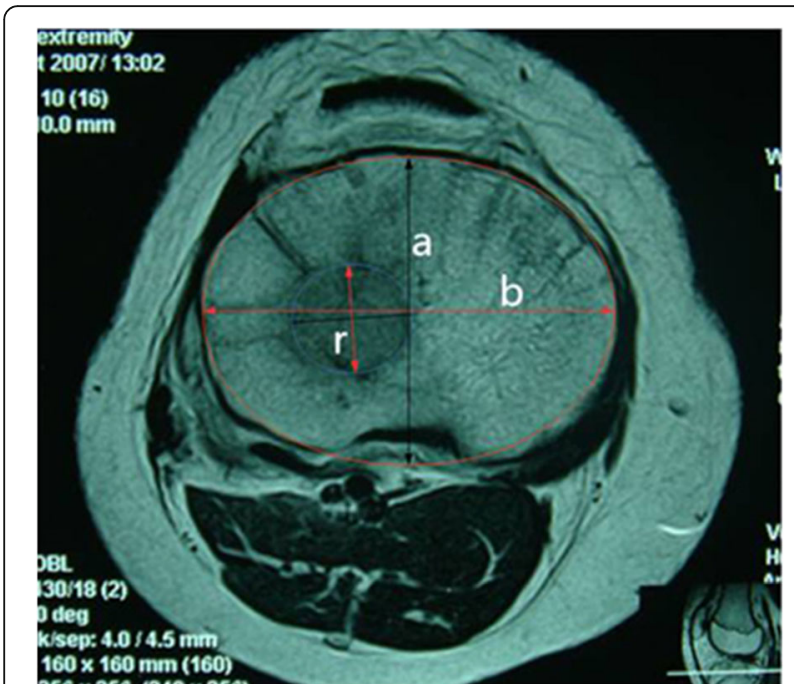

Fig. 2 The method of measuring the lesion epiphyseal plate. $r$ is the radius of the lesion, and $\mathrm{a}$ and $\mathrm{b}$ are minor and major axes of epiphyseal plate. Focus/epiphyseal plate $=\pi r^{2} / \pi a b$

\section{Surgical therapy}

Firstly, all patients have undergone meticulous intralesional curettage from the local cortical window and polished tumor cavity around the lesion edge, $2-5 \mathrm{~mm}$ by bone drill ( $2 \mathrm{~mm}$ of latent lesion, $3 \mathrm{~mm}$ of active, $5 \mathrm{~mm}$ of aggressive, Table 1). Secondly, tumor cavity was inactivated by $95 \%$ alcohol for $15 \mathrm{~min}$. Then, we used an electrotome to burn cavity in difficult-to-reach areas and saline solution to wash cavity repeatedly. Bone grafting was performed as follows. Allograft implantation was used in 13 patients, autologous iliac bone in 2 patients, and artificial bone (Medtronic, Inc., USA) in 5 patients. With the final processing, allogeneic bone block covered the cortical window, fixed with absorbable screw in seven patients and steel plate in a boy. There was no en bloc resection in this group. Patients were encouraged to perform a rehabilitation exercise with no weight bearing on the second day following surgery,

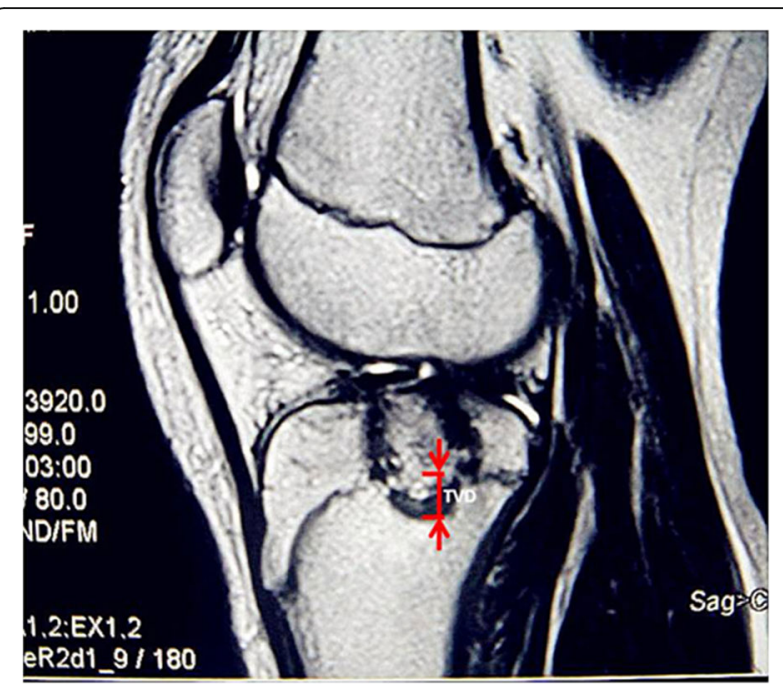

Fig. 4 TVD, the vertical dimension between the edge of lesion and epiphyseal line, was from the lower edge of the lesion to the epiphyseal line in the vertical plane of the sagittal or crown MRI plane. TVD is a negative data when the lesion is crossing the epiphyseal line

such as joint mobilization and muscle strength training. In the sixth week, patients started partial weight bearing and full-weight bearing at the third month following surgery. At 6 month after surgery, all patients qualified for social work and sports activity.

\section{Follow-up observations}

The follow-up occurred at $1,2,3,6,9,12$, and every 6 months thereafter. Imaging studies were focused on tumor recurrence and lesion limb growth and development. Local recurrence of the tumor was suspected if patient had persistent pain after surgery. We would carry out examination or MRI to exclude the pain from meniscus, cartilage, or soft tissue. Enlargement of the tumor on imaging studies, or bone marrow edema and cortical destruction on MRI, were thought to be signs of recurrence. Every 6 months after
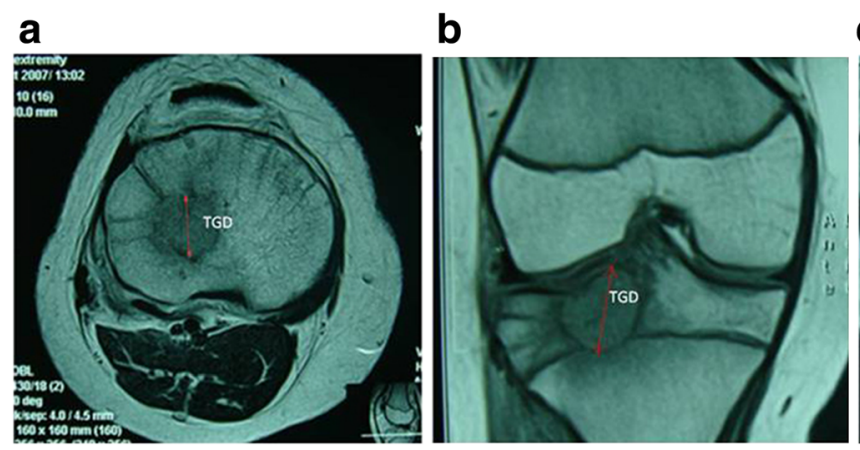

C

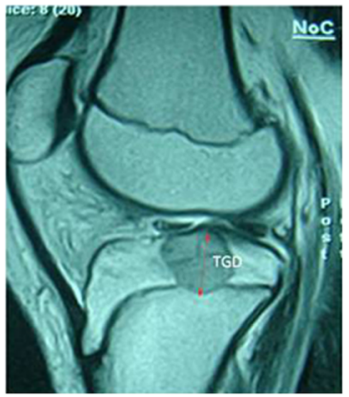

Fig. 3 TGD as the greatest dimension of the lesion was measured according to MRI in the crown, axial, or sagittal plane 
Table 2 The follow-up outcomes (mean \pm SD)

\begin{tabular}{lllllllll}
\hline & VAS & MSTS & ISOLS & \multicolumn{2}{l}{ SF-36 } & & & \\
\cline { 5 - 8 } & & & GH & PF & VT & RE & Soc \\
\hline Preoperation & $6.33 \pm 1.97$ & $23.67 \pm 1.46$ & None & $58.33 \pm 11.11$ & $58.61 \pm 11.48$ & $51.39 \pm 15.79$ & $55.56 \pm 10.13$ & $46.11 \pm 14.71$ \\
Last follow-up & $0.17 \pm 0.38^{*}$ & $28.56 \pm 1.34^{*}$ & $28.67 \pm 1.24$ & $91.11 \pm 4.39^{*}$ & $91.67 \pm 3.43^{*}$ & $88.61 \pm 5.37^{*}$ & $90.28 \pm 3.63^{*}$ & $89.17 \pm 5.22^{*}$
\end{tabular}

Abbreviations: VAS visual analog scale, MSTS93 Revised Musculoskeletal Tumor Society Rating Scale, SF-36 Medical Outcomes Study $36-$ Item Short-Form Health Survey, ISOLS International Society of Limb Salvage, GH general health, PF physical function, VT vitality, RE role emotional, Soc, social function

${ }^{*}$ Compared with preoperative, the differences were significant $(P<0.01)$

surgery, we scheduled a CT scan to master the status of bone grafting. The VAS scores, ISOLS grade, MSTS scores, and SF-36 scores were used to evaluate surgery effects. A comprehensive psychological intervention or treatment was performed in each followup. To explore the effect of surgical treatment on limb growth and development, we counted the shorten length of the lesion limb by X-ray examination and analyzed the relation between the shorten length with tumor activity, focus/epiphyseal plate, TGD, and TVD.

\section{Statistical analysis}

SPSS 19.0 (IBM Corporation, Armonk, NY, USA) software was used. The values are presented as mean \pm standard deviation (SD). Rank correlation was used to determine the relationship between the shorten length with tumor activity, focus/epiphyseal plate, TGD, and TVD. $r_{\mathrm{s}}$ was Spearman's rank correlation coefficient. Paired samples $t$ test was used to determine differences of VAS, MSTS93, and SF-36 between preoperation and the last follow-up. A $p$ value of less than 0.05 was considered significant.
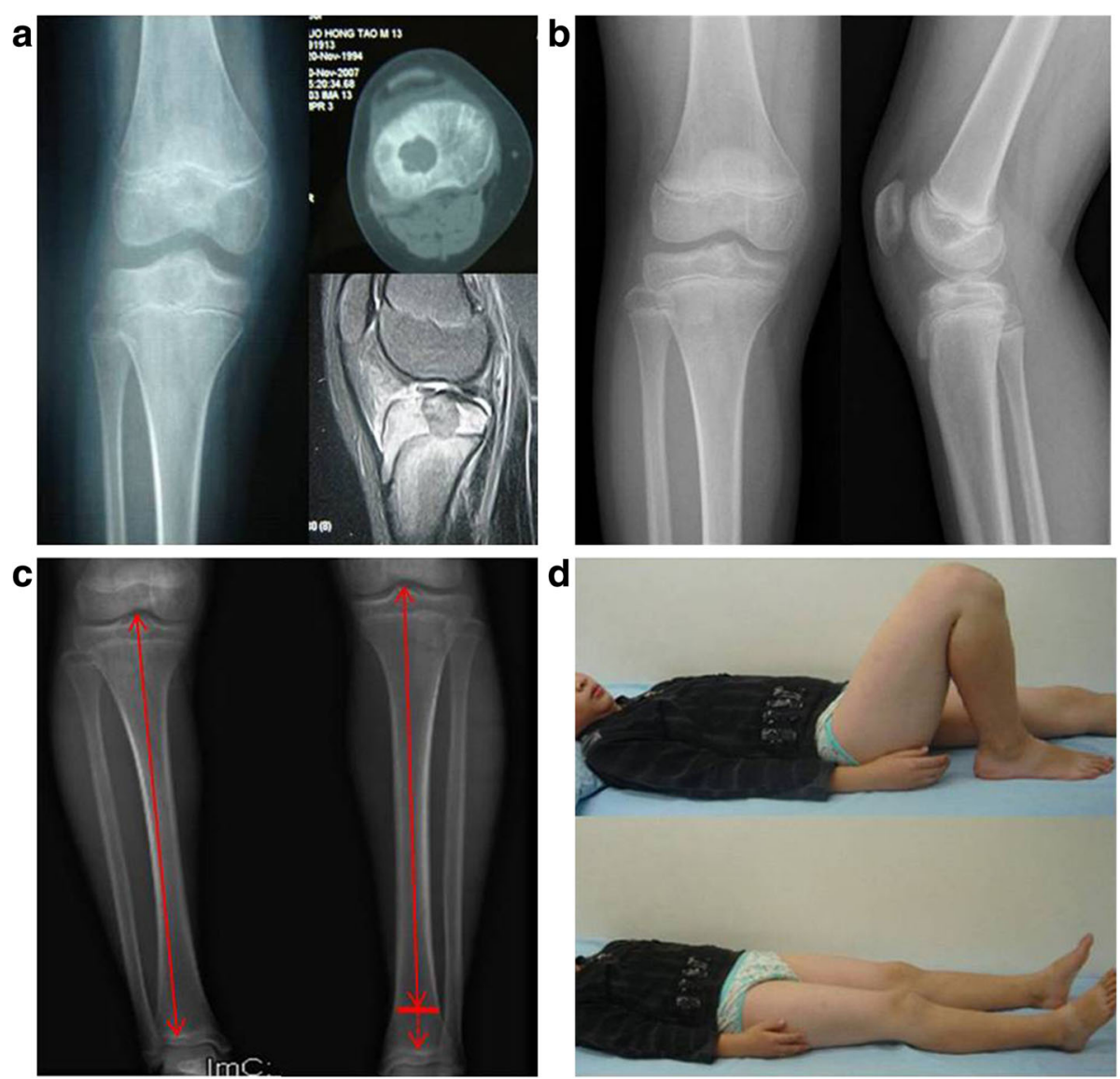

Fig. 5 a Preoperative radiographs of a 11-year-old girl. The radiolucent lesion (arrows) and bone cortical erosion in CT and MRI were visible in the proximal tibia. $\mathbf{b}$ and $\mathbf{c}$ were the X-rays of 3 and 5 years in the course of follow-up. We can see that the density of the tumor cavity become more and more high in the X-ray postoperative. Eight years later, the troubled limb was shorten by $14 \mathrm{~mm}$ compared with the healthy limb and mild varus deformity but had a satisfied function of the knee (d) 


\section{Results}

All patients were received follow-up with the median of $8.2 \pm 1.7$ years (5-11.5 years). The outcomes were summarized in Table 1. All wounds healed to grade A. No postoperative infections, delayed deep infection, nonspecific inflammation, rejection, allergies, hypersensitivity, and fractures were encountered. There was no evidence of local recurrence and distance metastasis in all cases. The patients' pain was completely resolved following surgery. There was no traumatic arthritis, joint collapse, or chronic joint pain in longterm follow-up. The ISOLS (International Society of Limb Salvage) functional grade was $28.67 \pm 1.24$ on average at the last follow-up. The function of MSTS93 and SF-36 have been significantly improved $(P<0.01)$ (Table 2, Figs. 5 and 6$)$. Eighteen patients have obtained excellent range of motion. Just an 11-year-old girl was observed with $5^{\circ}$ valgus deformity in the left tibia at 1-year anniversary. There was radiographic evidence of bone grafts completely incorporated postoperative 12 to 18 months.

The length of lesion limbs were shortened $18.47 \pm$ $7.22 \mathrm{~mm}(1.5-30 \mathrm{~mm})$ compared with non-surgery limb (Table 1). The shorten length have no obvious relativity with tumor activity $(P=0.061)$, but obvious relativity focus/epiphyseal plate $(P=0.015)$, TGD $(P=0.003)$, and TVD $(P=0.010)$ (Table 3$)$. In relation to the shorten length and TVD, $r_{\mathrm{s}}=-0.591$ was a negative correlation, which exposed that the bigger the value was, the lesser effective to the length shortening. In other words, the lesion crossing the epiphyseal line more led to more severe limb shortening (Fig. 7).

\section{Discussion}

Chondroblastoma is a benign tumor and mostly originates in an epiphyseal plate of long bone, which primarily occurs in children and adolescents. Previous studies reported first-line treatment should be lesion curettage $[1,9,12,13]$. Local recurrence rate of lesion curettage was 10 35\%. Risk factors of recurrence include location, young age, inadequate surgery, and biologic aggressiveness of tumor [12, 13]. Schreuder et al. reported that surgical technique might play the most important role in chondroblasoma recurrence [12]. Some reports showed that simple curettage was associated with higher recurrence rate because of too much worry about damaging an open epiphyseal plate [12, 13]. In this study, we preferred to use a bone drill to polish tumor cavity. With our experience, $2 \mathrm{~mm}$ of latent lesion, $3 \mathrm{~mm}$ of active, and $3-5 \mathrm{~mm}$ of aggressive were appropriate to remove residual tumor cells. We also took chemical (95\% alcohol) and thermal (electrotome) methods to inactivate tumor cavity. However, in areas near or crossing the epiphyseal line, we would open a small cortical window to remove tumor, which could minimize the epiphyseal line being injured. Besides, using a bone drill was relatively conservative, while chemical and thermal methods were more radical. Thus, those preprocessing techniques could prevent tumor recurrence drastically and reduce epiphyseal plate damage.

The epiphyseal plate is located between the epiphysis and metaphysis of long bones, which has complex anatomy with the following cellular layers: reserve
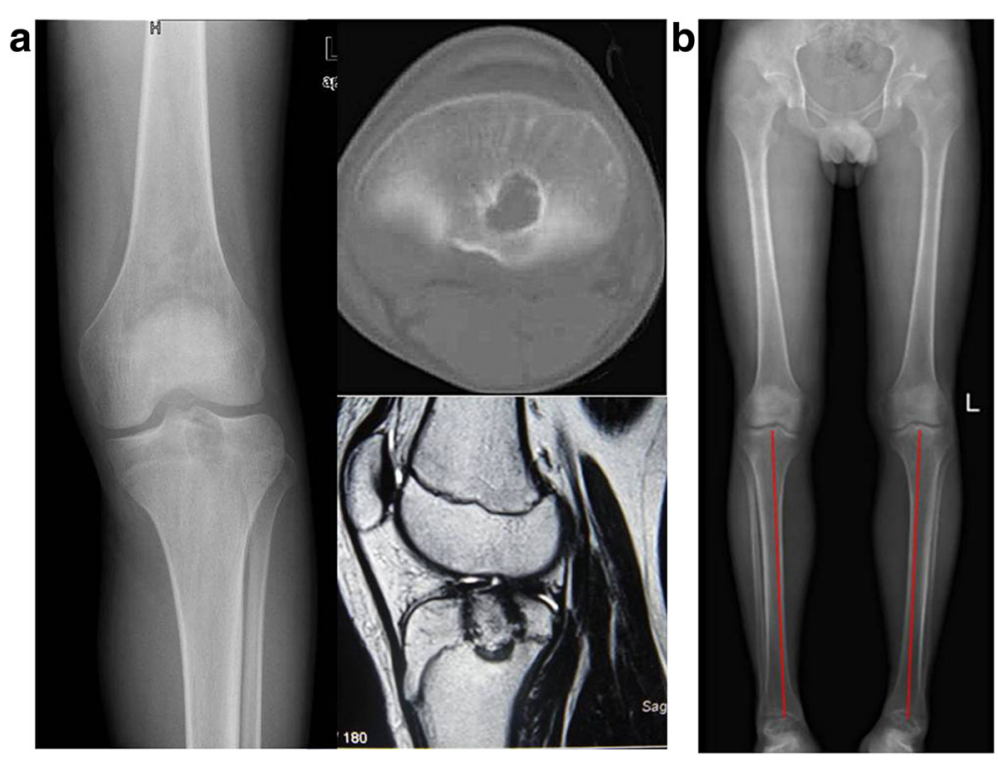

Fig. 6 A 13-year-old boy's X-ray, CT, and MRI (a) showed the lesion located at the proximal tibia. X-ray at 5.5 years postoperative told us that the grafting bone had been incorporated with the host bone and that the troubled limb shorten by $1.5 \mathrm{~mm}$ (b) compared with the healthy limb 
Table 3 Statistical data on 18 patients

\begin{tabular}{lll}
\hline & $r_{\mathrm{s}}$ & $P$ \\
\hline Tumor activity & 0.450 & 0.061 \\
TGD $(\mathrm{mm})$ & 0.665 & 0.003 \\
TVD $(\mathrm{mm})$ & -0.591 & 0.010 \\
Focus/growth plate & 0.565 & 0.015 \\
\hline
\end{tabular}

The negative value indicates that the lesion involves the epiphyseal plate; focus/growth plate, proportion between the largest area of the focus lesion in the horizontal and the epiphyseal plate. $r_{\mathrm{s}}$ was Spearman's rank correlation coefficient

Abbreviations: TGD the greatest dimension of the lesion, TVD the vertical dimension between the edge of lesion and epiphyseal line

zone, proliferative zone, and hypertrophic zone. It regulates endochondral maturation, degeneration, and calcification [14, 15]. The reserve zone is called the germinal or stem cell zone, which contains resting chondrocytes. The combination of chondrocyte proliferation, the enlargement of maturing chondrocytes in the hypertrophic zone, and the production of ECM (extracellular matrix) are the major contributors to longitudinal bone growth [16]. Tumor curettage may hurt the epiphyseal plate. However, the epiphyseal plate has limited ability to repair. Furthermore, vascular of metaphysis invaded into the broken epiphyseal plate and formed a fiber vascular bridge. Finally, a bone bridge was formed with a large number of calcium salt depositions, which is lacking of longitudinal growth ability, leading to limb shortening and angular deformity [17].

Previous studies have found that resistance of long bones to growth and development is related to the range of injured epiphyseal plate $[17,18]$. When the injured percentage of the central area is more than $7 \%$, the bone bridge formed and stretched the whole epiphyseal plate. Meanwhile, the eccentric lesion percentage could be more than $9 \%$, which caused obvious resistance $[19,20]$. In our study, all intralesion curettage led to epiphyseal plate injury, and many lesion areas were more than $10 \%$, one was up to $50 \%$. By follow-up, it resulted in limb shortening and deformity differentially as the body grows. Our outcome showed that focus/epiphyseal plate, TGD, and TVD were the key factors in a limb-growing capability disorder. In relation to shortened length and TVD, the lesion crossing the epiphyseal line more led to more severe limb shortening and
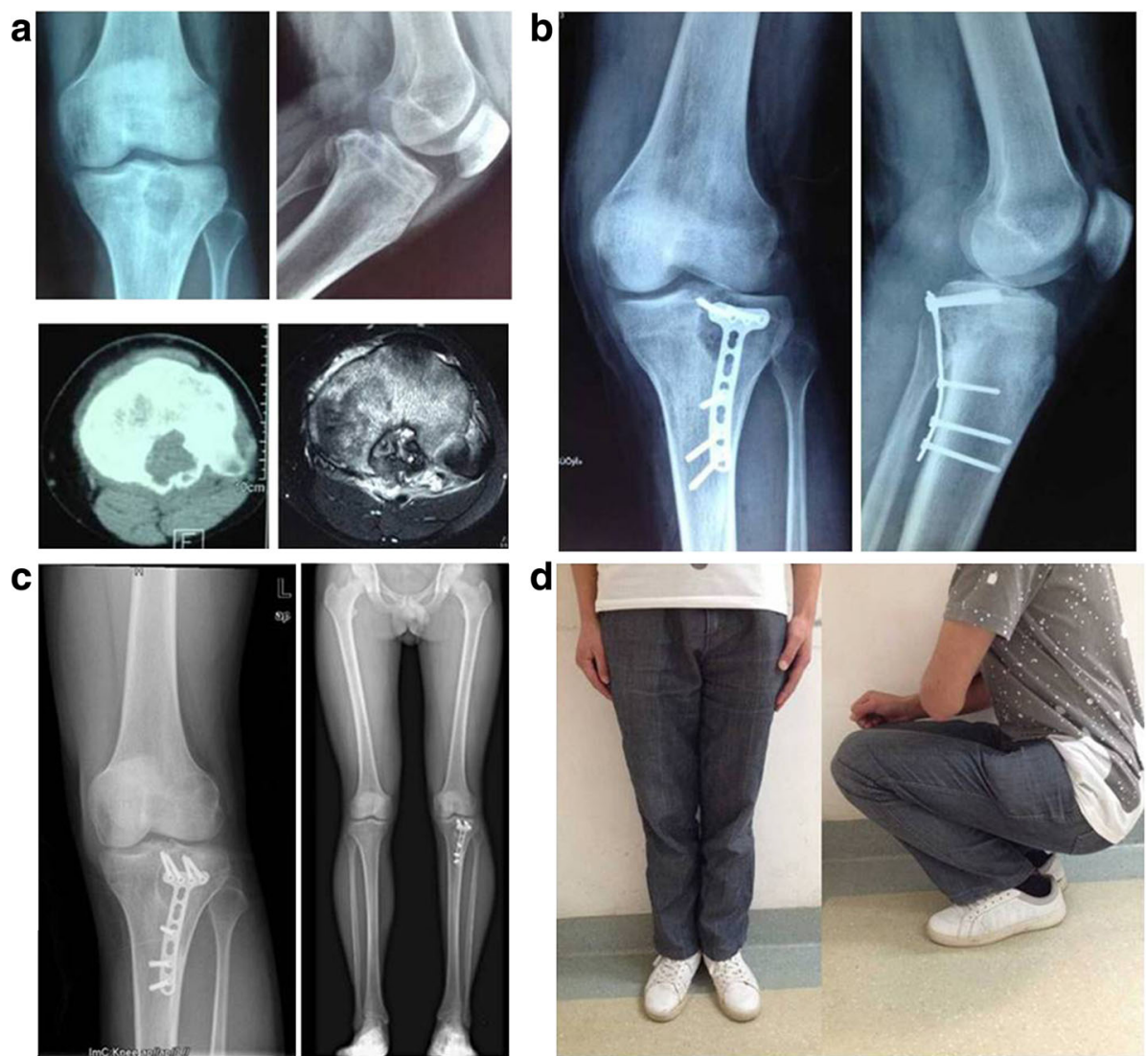

Fig. 7 A 15-year-old boy's X-ray, CT, MRI (a)showed the lesion located at posterior median of the proximal tibia. X-ray at 1 year(b) and 9.5 years(c) postoperative told us that the grafting bone had been gradually incorporated with the host bone and that the troubled limb shortened $20 \mathrm{~mm}$ compared with the healthy $\operatorname{limb}(\mathbf{d})$. The patient acquired a satisfactory function outcome and could run $3 \mathrm{~min} 43 \mathrm{~s}$ in $1-\mathrm{km}$ race 
deformity. In our series, the length of troubled limb were shorter $18.47 \pm 7.22 \mathrm{~mm}(1.5-30 \mathrm{~mm})$ compared with the healthy limb on 5 upper limbs and 13 lower limbs. Thirteen patients whose lesions were located in the lower limbs had no symptoms on walking because of a pelvic compensator, and the other five patients' shoulder function was satisfied at the last follow-up.

Limb length discrepancy and angular deformity may not necessarily lead to clinical problems during childhood and puberty, but psychosocial problems may occur. Social withdrawal, practical problems relating to clothing and shoes, fearing about future compatible partners, and career planning would suffer [21-23]. Lower limb length discrepancy would lead to posture deformation, gait asymmetry, low back pain, and discopathy [21, 22]. Thus, in addition to clinical problem improvement, it was necessary to make a comprehensive psychological intervening or treatment to guide patient's healthy and joyful growth. As we have known, leg length discrepancy $<2 \mathrm{~cm}$ is a static disorder. Leg length discrepancy $>3 \mathrm{~cm}$ causes distinct gait and posture disorders-the bigger the difference the greater and more distinct the disorders are [22]. In our study, all the patients underwent positive psychological intervention and clinical symptoms treatment after surgery. All patients' function of VAS, MSTS93, and SF-36 have been significantly improved $(P<0.01)$. They all joined in social activities in adulthood as farmer, construction worker, college students, civil servants, and teachers, and so on.

\section{Conclusion}

Managing of chondroblastoma located in an open epiphyseal plate of a long bone with meticulous curettage, inactivity, and bone grafts can control tumor progression and recurrence effectively. Meanwhile, early detection and prompt surgical treatment intervention, which reduced significantly the tumor to influence limb growth and development, get encouraging limb function.

\section{Limitation}

First, as a retrospective study, we had no control group to assess the superiority of our study compared with different tumor management. This was a retrospective review and is, therefore, limited by the heterogeneity of the available data and follow-up. Second, the sample size was few. We needed more cases and time to observe local recurrence, distant metastasis, limb discrepancy, and function. Furthermore, because of few cases in our study, we could not observe more factors, which might influence limb growth and development after surgical treatment, such as age and gender.
Funding

None

\section{Availability of data and materials}

The authors declare that all data supporting the findings of this study are available within the article.

\section{Authors' contributions}

$Y X, Y L, Z Y, H L$, and $X F$ participated in the collection of the clinical data and performed patients' follow-up. $Y X$ and $Y L$ drafted this manuscript. HD designed this research and reviewed the manuscript for important intellectual content. $\mathrm{CT}$ and $\mathrm{HD}$ were responsible for these patients' operation and participated in the project coordination and assisted with the manuscript. Each author has participated sufficiently in this work to take public responsibility for the appropriate portions of the manuscript. All authors read and approve of the final manuscript.

\section{Ethics approval and consent to participate}

This retrospective study has been approved by the ethics committee at West China Hospital of Sichuan University. Meanwhile, every patient had signed a consent about the treatment and study.

\section{Consent for publication}

Written consent for the publication of images used in the manuscript was obtained from the patients.

\section{Competing interests}

The authors declare that they have no competing interests.

\section{Publisher's Note}

Springer Nature remains neutral with regard to jurisdictional claims in published maps and institutional affiliations.

Received: 6 August 2017 Accepted: 10 January 2018

Published online: 23 January 2018

\section{References}

1. Lin PP, Thenappan A, Deavers MT, Lewis VO, Yasko AW. Treatment and prognosis of chondroblastoma. Clin Orthop. 2005;438:103-9.

2. Jaffe $H \mathrm{~L}$, Lichenstein $\mathrm{L}$. Benign chondroblastoma of bone. Am J Pathol. 1942;18(6):969-91.

3. Xu H, Nugent D, Monforte HL, Binitie TO, Ding Y, Letson GD, Cheong D, Niu $X$. Chondroblastoma of bone in the extremities: a multicenter retrospective study. JBJS. 2015;97(11):925-31.

4. Xie $C$, Jeys $L$, James SLJ. Radiofrequency ablation of chondroblastoma: longterm clinical and imaging outcomes. Eur Radiol. 2015;25(4):1127-34.

5. Ramappa AJ, Lee FY, Tang P, Carlson JR, Gebhardt MC, Mankin HJ. Chondroblastoma of bone. J Bone Joint Surg Am. 2000;82(2):1140-5.

6. Springfield DS, Capanna R, Gherlinzoni F, Picci P, Campanacci M. Chondroblastoma: a review of seventy cases. J Bone Joint Surg Am. 1985; 67(5):748-55.

7. Farfalli GL, Slullitel PAI, Muscolo DL, Ayerza MA, Aponte-Tinao L. What happens to the articular surface after curettage for epiphyseal chondroblastoma? A report on functional results, arthritis, and arthroplasty. Clin Orthop Relat Res. 2017:475(3):760-6.

8. Mashhour MA, Rahman MA. Lower recurrence rate in chondroblastoma using extended curettage and cryosurgery. Int Orthop. 2014;38(5):1019-24

9. Van Der Geest IC, Van Noort MP, Schreuder HW, Pruszczynski M, De Rooy JW, Veth RP. The cryosurgical treatment of chondroblastoma of bone: longterm oncologic and functional results. J Surg Oncol. 2007;96(3):230-4.

10. Lehner B, Witte D, Weiss S. Clinical and radiological long-term results after operative treatment of chondroblastoma. Arch orthop traum su. 2011; 131(1):45-52.

11. Patrick CT, Robert KHJ. General principles of tumors, Enneking system for staging benign and malignant musculoskeletal tumors. Campbell's operative orthopaedics, vol. 12; 2013. p. 791-2.

12. Schreuder HW, Pruszczynski M, Veth RP, Lemmens JA. Treatment of benign and low-grade malignant intramedullary chondroid tumours with curettage and cryosurgery. Eur J Surg Onco. 1998;24(2):120-6.

13. Tiefenboeck TM, Stockhammer V, Panotopoulos J, Lang S, Sulzbacher I, Windhager R, Funovics PT. Complete local tumor control after curettage of 
chondroblastoma—a retrospective analysis. Orthop Traumatol Surg Res. 2016;102(4):473-8.

14. Chung R, Xian CJ. Recent research on the growth plate: mechanisms for growth plate injury repair and potential cell-based therapies for regeneration. J Mol Endocrinol. 2014;53(1):T45-61.

15. Romain S, Frederick KW, Dietrich P. Surgical-experimental principles of anterior cruciate ligament (ACL) reconstruction with open growth plates. $J$ Exper Orthop. 2015;2(1):1-12.

16. Hamdy RC. Growth plate injuries: an introduction. Limb lengthening and reconstruction surgery case atlas: pediatric deformity; 2015. p. 83-6.

17. Xian CJ. Recent research on the growth plate: regulation, bone growth defects, and potential treatments. J Mol Endocrinol. 2014;53(1):E1-2.

18. San-julian M, JD Aquerreta AB, Caadell JL. Indications for epiphyseal preservation in metaphyseal malignant bone tumors of children relationship between image methods and histological findings. J Pediatr Orthop. 1999;19(4):543-8.

19. Janarv PM, Wikstrom B, Hirsch G. The influence of transphyseal drilling and tendon grafting on bone growth: an experimental study in the rabbit. J Pediatr Orthop. 1998;18(2):149-54.

20. Lee MA, Nissen TP, Otsuka NY. Utilization of a murine model to investigate the molecular process of transphyseal bone formation. J Pediatr Orthop. 2000;20(6):802-6.

21. Lecointre C, Toublanc JE. Psychological indications for treatment of tall stature in adolescent girls. J Pediatr Endocrinol Metab. 1997;10(5):529-31.

22. Raczkowski WJ, Daniszewska B, Zolynski K. Functional scoliosis caused by leg length discrepancy. Arch Med Sci. 2010;6(3):393-8.

23. D'Ambrosi R, Ragone V, Caldarini C, Serra N, Usuelli FG, Facchini RM. The impact of hereditary multiple exostoses on quality of life, satisfaction, global health status, and pain. Arch Orthop Trauma Surg. 2017;137(2):209-15.

\section{Submit your next manuscript to BioMed Central and we will help you at every step:}

- We accept pre-submission inquiries

- Our selector tool helps you to find the most relevant journal

- We provide round the clock customer support

- Convenient online submission

- Thorough peer review

- Inclusion in PubMed and all major indexing services

- Maximum visibility for your research

Submit your manuscript at www.biomedcentral.com/submit
Biomed Central 